\title{
émulations
}

\section{Revisiter la question du retour au prisme du transnationalisme, des trajectoires, des aspirations et des contraintes}

\author{
Marie-Laurence Flahaux
}

Émulations - Revue de sciences sociales 2020, n 34, «Transnationaliser le retour. Vers une révision du regard sur les migrations de retour contemporaines ».

\section{Article disponible à l'adresse suivante}

https://ojs.uclouvain.be/index.php/emulations/article/view/flahaux

\section{Pour citer cet article}

Marie-Laurence Flahaux, « Revisiter la question du retour au prisme du transnationalisme, des trajectoires, des aspirations et des contraintes », Émulations, $\mathrm{n}^{\circ} 34$, Mise en ligne le 7 octobre 2020.

DOI : 10.14428/emulations.034.08

Distribution électronique : Université catholique de Louvain (Belgique) : ojs.uclouvain.be

(C) Cet article est mis à disposition selon les termes de la Licence Creative Commons Attribution, Pas d'Utilisation Commerciale 4.0 International. http://creativecommons.org/licenses/by-nc/4.0/

Éditeur : Émulations - Revue de sciences sociales / Presses universitaires de Louvain https://ojs.uclouvain.be/index.php/emulations

ISSN électronique : 1784-5734

PUL PRESSES 


\title{
Revisiter la question du retour au prisme du transnationalisme, des trajectoires, des aspirations et des contraintes
}

Marie-Laurence Flahaux ${ }^{1}$

\begin{abstract}
[Résumé] Dans la conclusion, l'auteure propose une lecture personnelle des différentes contributions de ce numéro à travers un double exercice de mise en perspective théorique, en s'appuyant sur ses propres recherches sur les retours en contexte africain. Dans un premier temps, elle fait ressortir la manière dont les articles mettent en lien les concepts de « retour » et de «transnationalisme ». Dans un second temps, à partir d'éléments qui ont particulièrement retenu son attention, elle discute la façon dont la question du retour donne à voir (a) l'articulation entre les trajectoires familiales, matérielles, professionnelles et migratoires des migrants, (b) le décalage entre les aspirations des individus et les contraintes auxquelles ils font face, ainsi que (c) les stratégies qu'ils mettent en place face à ces dernières.
\end{abstract}

Mots-clés : retour, transnationalisme, trajectoires, contraintes, aspirations.

Revisiting the question of a return in the lens of transnationalism, trajectories, aspirations and constraints

[Abstract] In the conclusion, the author presents a personal reading of the contributions of this issue through a twofold exercise of theoretical perspective, drawing on her own research on return migration in the African context. Firstly, she highlights the way in which the articles link the concepts of « return » and « transnationalism ». Secondly, she discusses how the question of return reveals (a) the link between the family, material, professional and migratory trajectories of migrants, (b) the gap between the aspirations of individuals and the constraints they face, and (c) the strategies they implement to face the latter.

Keywords: return, transnationalism, trajectories, constraints, aspirations.

Les contributions de ce numéro portent chacune, à leur façon, sur la question du retour, celui-ci étant compris dans sa globalité et renvoyant à divers moments dans les trajectoires de vie des individus. À partir de méthodologies qualitatives et quantitatives, elles analysent la question du retour en se focalisant sur des temps plus ou moins longs dans les trajectoires individuelles. Ces temps sont marqués non seulement par des événements dans la vie des migrants, notamment sur le plan familial et matériel, mais aussi par l'évolution du contexte dans leurs pays d'origine et de destination. Les articles abordent les séjours temporaires au pays de migrants ouest-africains vivant en France, les semaines précédant le retour « volontaire » d'une Ivoirienne et de son fils au Maroc, les années que des Sénégalais ont passées en dehors de leur village depuis le moment de leur départ, la période pendant laquelle la question du retour s'est posée pour les Équatoriens s'étant retrouvés dans une Espagne en crise, et les années vécues

\footnotetext{
${ }^{1}$ Institut de recherche pour le développement, LPED, France.
} 
en France métropolitaine et dans les départements d'outre-mer pour les migrants qui y sont retournés. Dans ce numéro se retrouve donc une diversité de phénomènes analysés, d'approches utilisées, de trajectoires considérées, de perspectives adoptées, et de populations étudiées. Je me suis prêtée à deux exercices pour rédiger cette conclusion. Le premier a été de trouver un point commun aux diverses contributions. Chaque article, à sa manière, souligne le lien entre la question du retour et le transnationalisme. Dans un premier temps, ce lien sera discuté. Le deuxième exercice a été de faire le point sur ce qui a particulièrement retenu mon attention à la lecture des différentes contributions. Je détaillerai trois aspects dans un deuxième temps : l'articulation des différents types de trajectoires ou parcours de vie des migrants ; le décalage entre aspirations et capabilités (Sen, 1999) pour expliquer le ressenti des migrants face à leur situation en lien avec le retour; et les contraintes qui ne les empêchent pas de faire preuve d'agentivité.

\section{Le lien entre le transnationalisme et la question du retour}

Parmi les courants théoriques des migrations, le transnationalisme met en évidence le fait que les migrants, lorsqu'ils vivent à l'étranger, conservent des liens avec leur société d'origine (Levitt, Jaworsky, 2007 ; Levitt et al., 2003 ; Portes, 2003 ; Portes et al., 1999). Ce courant met surtout l'accent sur la façon dont les liens sont maintenus : en fréquentant les membres de la diaspora, à travers les visites qu'ils effectuent dans le pays d'origine, en faisant des transferts d'argent, ou en ayant des contacts avec des personnes restées au pays (Portes et al., 1999). Les activités transnationales peuvent donc concerner divers domaines (économique, politique, socioculturel, religieux) et varier en intensité (Levitt et al., 2003). En plus de contribuer à une meilleure compréhension des liens que les migrants entretiennent avec leur société d'origine, le transnationalisme permet de comprendre l'identité des migrants qui évolue au fur et à mesure du temps passé à l'étranger, et qui combine des traits à la fois de leur société d'origine et de la société de destination (Vertovec, 2001).

Le transnationalisme est utile pour mieux comprendre la question du retour. Il insiste sur la mobilité transnationale, sur les va-et-vient des migrants, sur leur identité d'« entre-deux », et semble considérer positivement la question du retour (Cassarino, 2004 ; Flahaux, 2013). D’abord, il suggère qu'il y a une relation positive entre liens transnationaux et intention du retour : les migrants qui ont des liens forts avec leur pays d'origine ont plus tendance à vouloir y retourner (Carling, Pettersen, 2014). Ensuite, il considère que le retour est préparé par les migrants via des visites régulières dans leur pays d'origine et par des contacts fréquents avec leur famille, leurs amis, et des membres de la diaspora, grâce aux nouvelles technologies. Ces liens leur permettent d'obtenir des informations sur l'évolution du contexte dans le pays d'origine, sur les opportunités qui y existent, et c'est sur cette base qu'ils prennent la décision de retourner. Enfin, le transnationalisme suppose que la préparation du retour, grâce aux liens maintenus avec le pays d'origine, a un effet positif sur la réinsertion des migrants 
après leur retour (Cassarino, 2004). Le transnationalisme semble donc considérer que les liens transnationaux jouent en faveur du projet et de la décision du retour et qu'ils influencent positivement la réinsertion après le retour. Les contributions de ce numéro viennent apporter des compléments et des nuances à cette lecture du lien entre le transnationalisme et la question du retour.

Pour commencer, les contributions suggèrent que les liens transnationaux avec le pays d'origine ne vont pas toujours de pair avec une intention de retourner ou la réalisation d'un retour. L'article d'Anna Perraudin montre que le transnationalisme, appréhendé à travers les biens immobiliers dans lesquels les migrants ont investi dans leur pays d'origine pendant leur migration, peut aussi être utilisé dans l'idée d'éviter le retour. Dans le contexte de l'Espagne en crise, elle met en évidence que les biens immobiliers en Équateur constituent un capital économique que les migrants équatoriens mobilisent afin de s'installer, si pas définitivement, en tout cas plus longuement en Espagne, ou pour migrer ailleurs. Avec la contribution de Césarine Sambou, qui analyse les retours temporaires - une pratique transnationale par nature - de migrants ouest-africains résidant en France, notamment au Sénégal et au Burkina Faso, on comprend que les retours de plus long terme ne sont pas envisagés par ces derniers. Elle dévoile l'expérience difficile de ces visites pour les migrants qui n'ont pas les moyens de répondre aux attentes des membres de leur famille restés au pays. En raison de leur refus et de leur incapacité à répondre aux sollicitations, ils font l'objet de stigmatisations et de dévalorisations. L’identité transnationale de ces migrants influence non seulement leur perception de leur société d'origine, mais aussi la façon dont ils sont eux-mêmes perçus, à savoir comme des personnes ayant une manière occidentale de vivre et qui ne partagent pas les richesses accumulées. Bien que l'article n'aborde pas la question du retour de longue durée ${ }^{2}$, on peut en déduire que les migrants savent qu'un retour, qu'il soit court ou long, requiert une certaine préparation, et que ceux qui ont vécu une expérience difficile lors d'un retour temporaire n'ont pas l'intention de revenir s'installer durablement dans leur pays d'origine. Les liens transnationaux renvoient aux attentes de la famille. Cet aspect est présent dans plusieurs contributions. Samuel Lietaer, Loïc Brüning et Coumba Ndoffene Faye soutiennent que, pour les Sénégalais des zones rurales, le retour est souvent négocié entre ces derniers et leur famille restée au village, surtout lorsque le choix de partir en migration a résulté d'une stratégie collective. Par conséquent, les migrants ne sont pas incités à revenir, la famille ne souhaitant pas voir s'arrêter les transferts de fonds dont elle bénéficie. Les attentes de la famille en termes de transferts de fonds des migrants expliquent donc que les migrants n'envisagent pas de se réinstaller dans les villages d'origine et que, dans les faits, rares sont ceux qui retournent de façon permanente, en particulier dans les zones où les décisions migratoires sont prises collectivement. Les pratiques transnationales telles que les transferts d'argent et les visites de courtes durées n’influenceraient donc

\footnotetext{
${ }^{2}$ Les retours de longue durée sont appelés « retours » dans ce texte, et les retours de courte durée « visites ».
} 
pas toujours positivement le retour. Mes recherches sur les déterminants des retours pour les Sénégalais qui ont migré en Europe montrent aussi que les pratiques transnationales peuvent se présenter comme une alternative au retour. Elles indiquent que ceux qui ont fait au moins une visite au pays depuis leur départ sont moins susceptibles d'effectuer un retour de longue durée que ceux qui ne sont jamais retournés (Flahaux, 2017). Les migrants qui retournent au Sénégal, en fait, ont plus de chances de ne jamais avoir effectué de visite pendant la migration. Ceux qui retournent sont souvent partis en Europe avec l'idée d'atteindre un objectif, et ils n'effectuent pas de visite au pays afin de pouvoir épargner et d'éviter certains coûts (relatifs aux frais de voyage, mais aussi de cadeaux à l'entourage, et de sollicitations sur place, comme l'a montré Césarine Sambou). Ainsi, dans certains cas, les pratiques transnationales ont pour effet d'aider les migrants à ne pas retourner et, dans d'autres cas, elles se présentent davantage comme une alternative au retour.

Ensuite, deux autres contributions permettent de comprendre que l'effet du transnationalisme sur la question du retour peut dépendre du profil et des trajectoires des migrants ainsi que du type de liens transnationaux. D'une part, la contribution de Marine Haddad me conduit à penser que les liens maintenus avec le lieu d'origine peuvent influencer les trajectoires migratoires des individus en lien avec le retour, mais différemment selon le profil des migrants. La comparaison entre hommes et femmes migrant·e.s originaires des départements d'outre-mer à laquelle elle procède suscite des réflexions en ce sens. Elle montre que les hommes sont moins souvent contraints de retourner, et qu'ils se mettent en couple et deviennent pères assez rapidement après leur retour. Cette trajectoire des hommes reflète un certain succès de la migration et du retour, et elle contraste avec celle des femmes, pour qui le retour non seulement retarde la mise en couple ainsi que la naissance d'enfants, mais aussi fragilise les unions qui étaient en cours, surtout lorsque le retour est associé à des contraintes et à des difficultés. Il est possible que les femmes aient entretenu moins de liens avec le lieu d'origine, ou que ces liens soient par nature différents de ceux maintenus par les hommes, ce qui peut avoir un impact sur la décision du retour et le devenir après le retour. Les pratiques transnationales des migrants en fonction de leur profil ainsi que leur lien avec les trajectoires de vie et la question du retour mériteraient d'être approfondis dans des recherches futures. D'autre part, Anissa Maâ, à travers son analyse fine du processus de décision de retour « volontaire » d'une jeune Ivoirienne qui se trouve au Maroc avec son nouveau-né, met en évidence les différents types de liens transnationaux qu'a cette dernière pendant la période qui précède son retour. Hésitante à l'idée de retourner, c'est lorsqu'elle apprend via les réseaux sociaux l'infidélité du père de son enfant au pays qu'elle se décide à retourner en Côte d'Ivoire afin de retrouver " sa place » auprès de cet homme. Cependant, un lien transnational d'un autre type vient retarder son retour ; il s'agit du service consulaire à l'ambassade de Côte d'Ivoire, qui refuse de reconnaître la validité de l'extrait de naissance marocain du nouveau-né. Les démarches qui s'en suivent sont longues avant que la jeune femme puisse obtenir 
le formulaire qui servira à l'émission du laissez-passer de son fils. Enfin, elle mobilise un autre type de ressource transnationale, son passeur, afin de régler ses comptes en récupérant l'argent qu'il lui doit et de pouvoir préparer son retour. On voit donc que les différents liens ont des effets sur la décision du retour et sur le moment de ce retour, certains l'accélérant et d'autres le retardant. L'influence des différents types de liens, de pratiques et de ressources transnationales sur les trajectoires de vie des migrants, en lien avec la question du retour, pourrait également être analysée en profondeur dans des recherches ultérieures.

\section{2. Étudier la question du retour à partir des trajectoires, des aspirations et des contraintes}

La lecture des contributions de ce numéro a retenu mon attention à trois niveaux. En premier lieu, les articles mettent en évidence l'articulation entre les trajectoires de vie, familiales, matérielles, professionnelles et migratoires des individus. Dans ses analyses sur les ressortissants des départements d'outre-mer, Marine Haddad examine comment les histoires migratoires et familiales des migrants qui sont retournés s'articulent. De façon originale et intéressante, elle démontre que les calendriers familiaux et migratoires s'organisent selon des logiques de genre. Cela donne envie d'en savoir plus sur d'autres aspects des histoires de vie de ces migrants et migrantes, notamment sur les plans professionnel, économique et transnational, car ces histoires peuvent également avoir un impact sur la décision du retour et le devenir après le retour. Anna Perraudin met en évidence que, pour les Équatoriens qui ont migré en Espagne, les stratégies migratoires concernant le retour et les stratégies immobilières sont connectées, et qu'elles sont prises en tenant compte de la famille. Anissa Maâ, qui se penche sur le parcours d'une migrante ivoirienne au Maroc avant son retour, explique comment le fait d'attendre un enfant au pays l'a poussée à prendre les choses en main pour organiser sa migration, et comment l'arrivée de son enfant (au Maroc) la conduit à ne pas monter sur une embarcation pour traverser la Méditerranée et rejoindre l'Europe. Ainsi, les événements familiaux peuvent accélérer ou venir perturber les parcours que les migrants avaient envisagés initialement. Je trouve intéressant de prendre en considération l'articulation des différentes trajectoires de vie pour mieux comprendre les éventuels projets de retour, les décisions de retourner et ce que deviennent les migrants après un premier retour dans leur pays d'origine. Ces trois phénomènes peuvent d'ailleurs être liés, raison pour laquelle une approche « englobante » de la question du retour a du sens (Flahaux, 2013). À ce propos, dans un article récent, je suggère que la question de la réinsertion consécutive au retour ne se pose pas seulement après le retour, mais aussi avant, car c'est sur base de l'anticipation de leur réinsertion en cas de retour au pays que les migrants planifient (ou non) de retourner et qu'ils retournent (ou non) (Flahaux, 2020).

En deuxième lieu, j’ai été très intéressée par ce que Anissa Maâ qualifie de « décalage entre espérances et chances» - termes empruntés à Pierre Bourdieu (2015) - et 
qui génère un sentiment d'insatisfaction chez les individus. Cela renvoie au décalage entre " aspirations » et « capabilités » (Sen, 1999), termes qui ont aussi été repris pour expliquer la migration ou l'immobilité (Carling, 2002 ; de Haas, 2011), mais qui peuvent être appliqués à d'autres phénomènes. Dans ce numéro, ce décalage entre espérances/ aspirations d'une part et chances/capabilités d'autre part ressort de plusieurs contributions. Après avoir expliqué le parcours d'Aya, qui aspirait à donner à son enfant le meilleur avenir possible mais qui s'est retrouvée dans l'impossibilité de rejoindre l'Europe, et qui espérait réussir socialement malgré son retour en Côte d'Ivoire dans des conditions précaires, Anissa Maâ écrit : «Peu importent ses choix, Aya voit donc continuellement ses aspirations contredites par les possibilités objectives de leur réalisation » (page 68 de ce numéro). Césarine Sambou, quant à elle, montre que les migrants ouest-africains effectuent une visite dans leur pays d'origine parce qu'ils souhaitent continuer à être considérés comme faisant partie de la famille et satisfaire les attentes de celle-ci. Cependant, un décalage apparaît entre leurs aspirations et les ressources limitées dont ils disposent, ce qui peut engendrer un sentiment d'exclusion et de marginalisation. Anna Perraudin indique aussi qu'il y a des cas où, bien que souhaité, le retour en Équateur est remis à plus tard en raison de la crise en Espagne et des dettes contractées, ce qui peut donner lieu à des sentiments de frustration pour les migrants. Il en est de même pour les étudiants mentionnés par Sylvie Bredeloup dans son entretien : rentrés au Burkina Faso après plusieurs années passées dans les universités islamiques d'Afrique et d'ailleurs, ils rencontrent de grandes difficultés pour se réinsérer professionnellement du fait que leurs diplômes ne sont pas reconnus et ne peuvent pas être valorisés après le retour. S’atteler à mieux comprendre le vécu et le ressenti des migrants dans leur expérience en lien avec la question du retour à partir du décalage entre leurs aspirations (ou espérances, désirs, espoirs, souhaits ou besoins) et les contraintes auxquelles ils font face est une autre piste à poursuivre.

En troisième lieu, l'agentivité ou « agency » des migrants apparaît clairement dans plusieurs des articles lorsque sont abordés les obstacles et contraintes qu'ils rencontrent. Face à l'incertitude, les migrants ne restent pas passifs ; ils mettent en place des stratégies. Pour les migrants qui savent qu'ils vont devoir faire face aux attentes exigeantes de la famille lors de leurs visites au pays, explique Césarine Sambou, il va s'agir de ne pas rester trop longtemps au pays et de vivre dans un logement séparé de celui de la famille. Pour les migrants équatoriens touchés par la crise en Espagne et ayant des enfants qui ne veulent pas quitter l'Espagne, écrit Anna Perraudin, il va s'agir de revoir leurs ambitions de retour en s'installant définitivement en Espagne grâce aux investissements dans l'immobilier qu'ils ont réalisés en Équateur. Anissa Maâ développe aussi les stratégies qu'élabore la migrante ivoirienne face à tous les blocages et à l'incertitude caractérisant l'aventure marocaine. Elle explique par exemple que la jeune femme décide de prolonger son séjour au Maroc et ne se présente pas à la première opportunité de retour que lui offre l'Organisation internationale pour les migrations (OIM), afin de mieux s'armer face aux risques de précarisation qu'induit son retour. Des recherches 
sur la question du retour mériteraient d'être poursuivies en mobilisant le cadre de « structure » et « agency » tel que le préconisait Oliver Bakewell (2010) pour les études sur les migrations en général. Elles pourraient apporter des éléments intéressants et utiles pour mieux comprendre comment les migrants se projettent dans l'avenir et développent des stratégies en vue d'un retour (ou non), pour se réinsérer après le retour, ou encore pour circuler, tout en tenant compte des contraintes et des risques auxquels ils font face ou qu'ils sont susceptibles de rencontrer.

\section{Bibliographie}

BaKeWell O. (2010), « Some Reflections on Structure and Agency in Migration Theory », Journal of Ethnic and Migration Studies, vol. 36, n 10, p. 1689-1708.

Bourdieu P. (2015), Méditations pascaliennes, Paris, Points.

CARLING J. (2002), "Migration in the Age of Involuntary Immobility: Theoretical Reflections and Cape Verdean Experiences », Journal of Ethnic and Migration Studies, vol. 28, n 1, p. 5-42.

Carling J., Pettersen S. (2014), "Return Migration Intentions in the Integration Transnationalism Matrix », International Migration, vol. 52, n 6, p. 13-30.

Cassarino J.-P. (2004), "Theorising Return Migration: the Conceptual Approach to Return Migrants Revisited », International Journal on Multicultural Societies, vol. 6, $\mathrm{n}^{\circ}$ 2, p. 253-279.

DE HAAS H. (2011), «The Determinants of International Migration. Conceptualizing Policy, Origin and Destination Effects", International Migration Institute Working Paper, vol. 32. En ligne, consulté le $1^{\text {er }}$ juillet 2020. URL : https://www.migrationinstitute. org/publications/wp-32-11.

Flahaux M.-L. (2013), Retourner au Sénégal et en RD Congo. Choix et contraintes au cœur des trajectoires de vie des migrants, Louvain-la-Neuve, Presses universitaires de Louvain.

Flahaux M.-L. (2017), « Home, Sweet Home? The Effect of Return Migration Support Mechanisms on Reintegration », Espace Populations Sociétés, $n^{\circ} 1$. En ligne, consulté

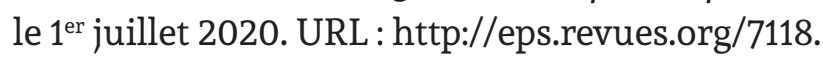

Flahaux M.-L. (2020), « Reintegrating after Return: Conceptualisation and Empirical Evidence from the Life Course of Senegalese and Congolese migrants », International Migration. En ligne, consulté le $1^{\text {er }}$ juillet 2020. DOI : https://doi.org/10.1111/ imig.12705.

Levitt P., DeWind J., Vertovec S. (2003), « International Perspectives on Transnational Migration: An Introduction », International Migration Review, vol. 37, n 3, p. 565-575.

Levitt P., Jaworsky B.N. (2007), « Transnational Migration Studies: Past Developments and Future Trends », Annual Review of Sociology, vol. 33, n 1, p. 129-156. 
Portes A. (2003), « Conclusion: Theoretical Convergencies and Empirical Evidence in the Study of Immigrant Transnationalism », International Migration Review, vol. 37, $n^{\circ} 3$, p. 874-892.

Portes A., Guarnizo L.E., Landolt P. (1999), « The Study of Transnationalism: Pitfalls and Promise of an Emergent Research Field », Ethnic and Racial Studies, vol. 22, n ${ }^{\circ}$, p. 217-237.

SEN A. (1999), Development as Freedom, New York, Anchor Books.

Vertovec S. (2001), « Transnationalism and identity », Journal of Ethnic and Migration Studies, vol. 27, n 4, p. f573-582. 